\title{
Renal Metastasis of Small Cell Lung Cancer With Urothelial Carcinoma of the Bladder Misdiagnosed as Renal Cell Carcinoma
}

\author{
Jin Young Lee ${ }^{\mathrm{a}}$, Jehun Kima, b
}

\begin{abstract}
Metastases to the kidney from lung cancer are very rare and are usually therefore recognized at autopsy. A 71-year-old man visited the urology department due to symptoms of worsening hematuria. An abdominal computed tomography scan showed a right renal mass. After a nephrectomy, he was diagnosed with small cell lung carcinoma, and further investigation showed small cell lung cancer in the central area of the left upper lobe with hepatic, bone and brain metastases. But his hematuria was unchanged. Therefore, we additionally diagnosed urothelial cell carcinoma in the bladder by transurethral resection of the bladder tumor. He was treated with etoposide and cisplatin chemotherapy. This is a unique antemortem case report in the literature of small cell lung cancer metastasizing to the contralateral kidney, liver, bone and brain diagnosed concurrently with urothelial cell carcinoma in the bladder.
\end{abstract}

Keywords: Hematuria; Lung cancer; Urothelial carcinoma; Kidney

\section{Introduction}

Small cell carcinoma (SmCC) commonly occurs in the lung, but may develop in extrapulmonary sites such as the head and neck, gastrointestinal tract, pancreas, gallbladder, uterine cervix, prostate and kidney [1]. Small cell lung cancer (SCLC) metastases can appear in almost any organ. It is difficult to distinguish renal metastases from primary renal cell carcinoma without histopathology, although this is an important distinction. Renal metastases from SCLC are uncommon, even though kidney metastases from lung primaries are frequently found postmortem [2].

Here, we describe a case of SCLC disseminating to the

Manuscript submitted August 12, 2019, accepted August 20, 2019

aDepartment of Internal Medicine, Kosin University Gospel Hospital, Kosin University College of Medicine, Busan, Korea

${ }^{b}$ Corresponding Author: Jehun Kim, Department of Internal Medicine, Kosin University College of Medicine, 262, Gamcheon-ro, Seo-gu, Busan 602-702, Korea. Email: libertier@gmail.com

doi: https://doi.org/10.14740/jmc3353 kidney, liver, bone and brain along with urothelial carcinoma that was diagnosed antemortem.

\section{Case Report}

A 71-year-old man presented with a 4-month history of worsening dysuria and hematuria. He was a bus driver and a heavy smoker (60-pack-year). An enhanced abdominal computed tomography (CT) scan showed a $2.6 \mathrm{~cm}$ irregular and ill-defined heterogeneous contrast-enhanced lesion in the right upper pole (Fig. 1). We suspected renal cell carcinoma, so the patient underwent right laparoscopic radical nephrectomy (Fig. 2). Pathologic examination after surgery revealed SmCC. Immunohistochemical staining was positive for CD56, synaptophysin, chromogranin and TTF-1, and negative for Ki67 and P53 (Fig. 3). Although the operation was performed successfully, his hematuria continued. Flexible cystoscopy demonstrated a bladder mass. A transurethral resection of the bladder tumor was undertaken, and histology was consistent with invasive, high-grade urothelial carcinoma (Fig. 3). The patient was evaluated with a positron emission tomography/computed tomography (PET/CT) scan

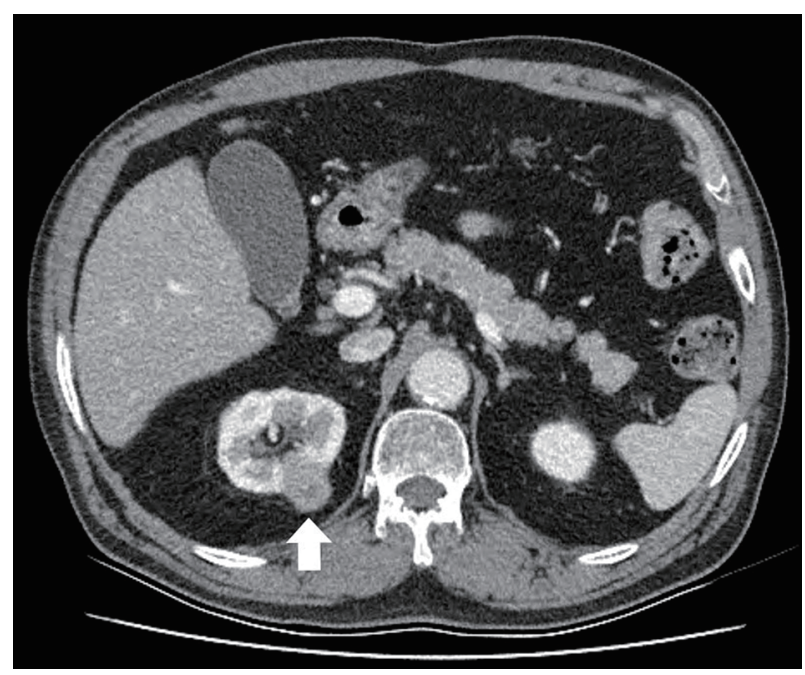

Figure 1. An abdominal computed tomography scan reveals the right renal mass. The tumor is seen as an irregular and ill-defined heterogeneous contrast-enhanced lesion. 


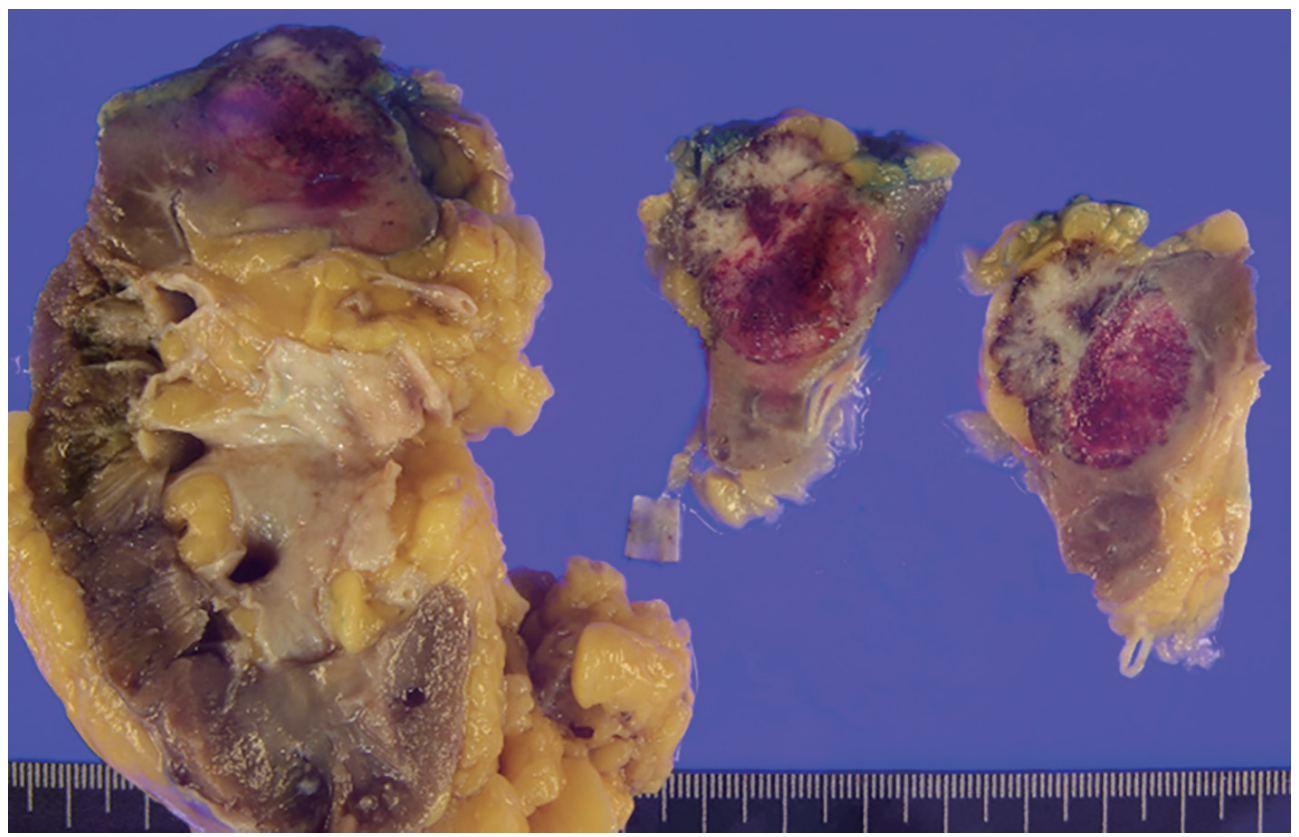

Figure 2. Gross picture of the excised renal tumor and kidney. The tumor directly invades the perirenal fat, but not beyond Gerota's fascia (pT3a).
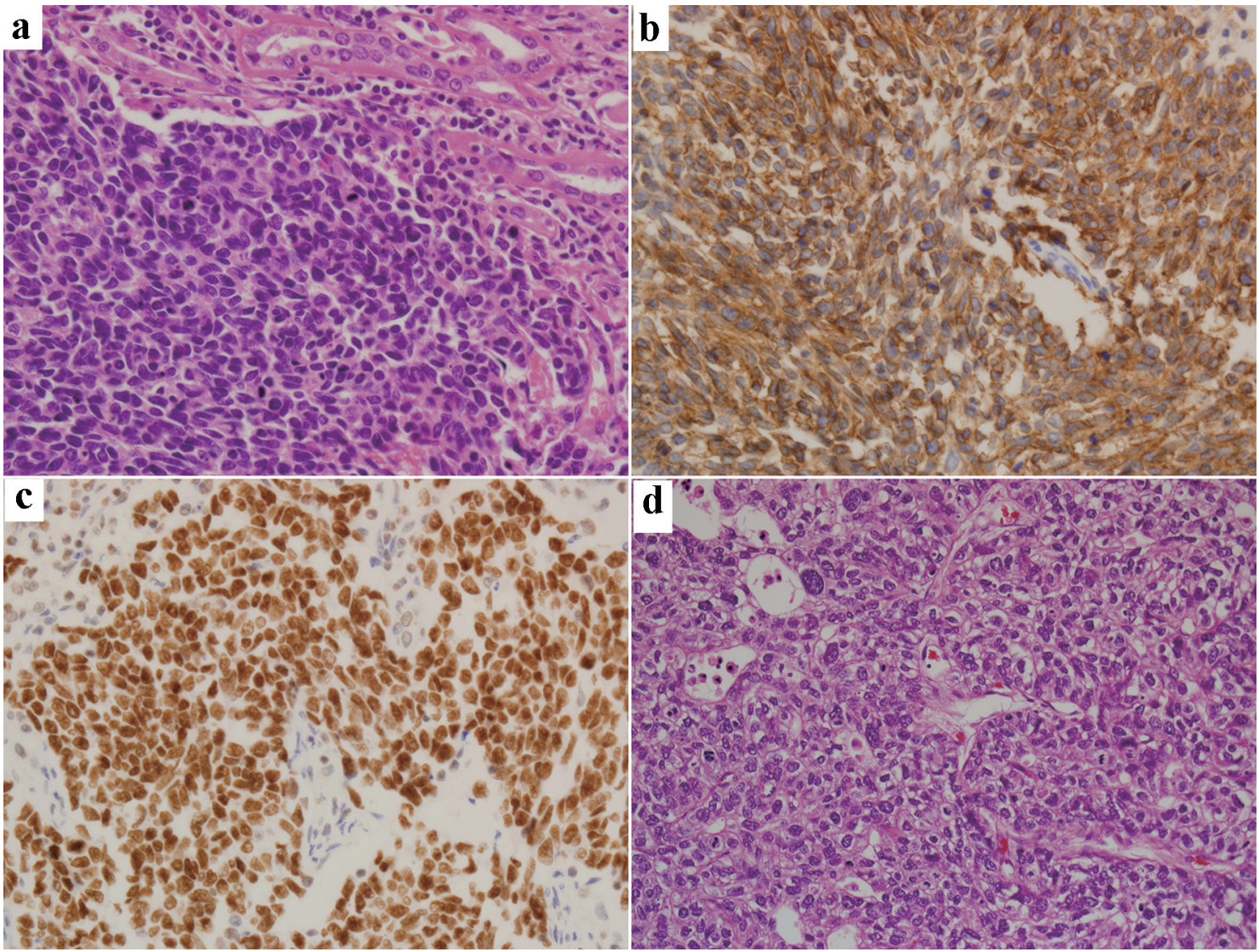

Figure 3. Renal biopsy specimen (original magnification, $\times 400$ ): hematoxylin-eosin staining (a) and immunohistochemical staining of CD56 (b), TTF-1 (c) and bladder biopsy specimen ( $\times 400)$ : hematoxylin-eosin staining (d). 


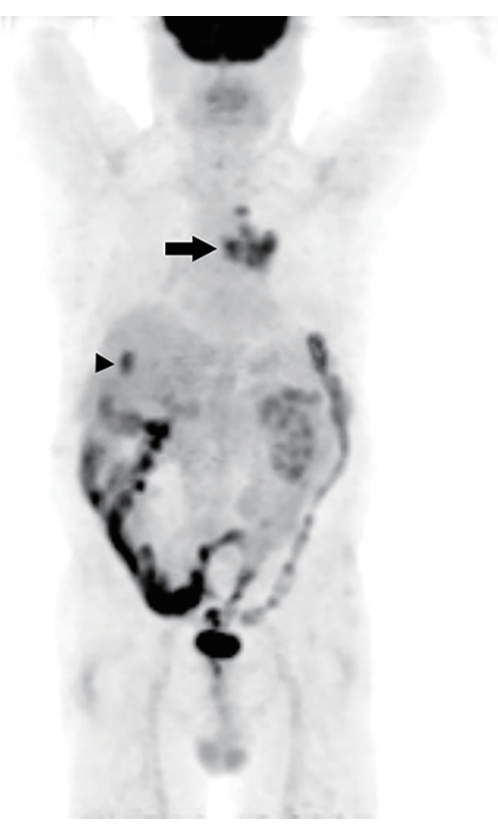

Figure 4. Maximum intensity projection (MIP) image from an positron emission tomography/computed tomography (PET/CT) scan with $18 \mathrm{~F}$ fluorodeoxyglucose (F18 FDG) shows intense uptake in the lung mass invading the central left upper lobe (arrow) and an oval focus of intense uptake in the right hepatic lobe (arrowhead). Post-operation change shows diffuse uptake in the right lower quadrant of the abdominal wall.

with 18F-fluorodeoxyglucose (F18 FDG), which showed significant uptake within the central left upper lobe, left hilar and left lower paratracheal lymph nodes, the aortopulmonary (AP) window, the right hepatic lobe, the $\mathrm{C} 7$ vertebral body and the right lower quadrant (RLQ) abdominal wall (Fig. 4). Therefore, we performed an endobronchial ultrasoundguided transbronchial needle aspiration (EBUS-TBNA) of the left paratracheal lymph node and subcarinal lymph node. This procedure confirmed the diagnosis of SCLC, and we observed the same positive tumor biomarkers as seen in the surgical kidney samples. A brain magnetic resonance imaging (MRI) showed multiple enhancing nodular metastatic lesions. The final diagnosis was SCLC metastasizing to the contralateral kidney, liver, lymph nodes, bone and brain along with urothelial carcinoma of the bladder. Our patient will receive six courses of combination chemotherapy using $100 \mathrm{mg} / \mathrm{m}^{2}$ etoposide and $60 \mathrm{mg} / \mathrm{m}^{2}$ cisplatin.

\section{Discussion}

This patient had SCLC that metastasized to the kidney, liver, lymph nodes, bone and brain along with urothelial bladder cancer, and histology was confirmed by nephrectomy and EBUSTBNA. To the best of our knowledge, there are few cases of these concurrent tumors reported in the literature.

SCLC comprises $20-25 \%$ of all lung cancers and is characterized by a swift clinical course and metastasis [3]. Tumor dissemination is a process that includes shedding, penetration, migration and proliferation of the primary tumor lesion. The anatomic structure of the primary tumor, the inherent anatomical structure of the target lesion and other elements can affect tumor production of metastases in the target organs [4].

The patient is a heavy smoker and a CT scan showed a 3 $\mathrm{cm}$ sized small, solid, enhancing renal mass with perinephric fat involvement. Such lesions are usually suspected to be renal cell carcinoma, although the management of small renal masses is controversial [5]. Through a surgical operation, we confirmed that the diagnosis was SmCC from the lung. SmCCs are mostly found in the lungs, but may occur in a wide variety of organs [1]. Renal SmCC is more uncommon than SCLC, which should always be considered when examining renal SmCC. An abdominal CT scan is a useful tool to screen for renal tumors. However, on CT scan, renal metastases resemble renal cell carcinoma, transitional cell carcinoma, renal abscesses and renal sarcoma. CT images of renal metastases were characterized as small, multiple, bilateral, wedge-shaped, less exophytic, and located within the renal capsule [6].

Our patient's hematuria persisted despite laparoscopic radical nephrectomy. Patients with renal metastases usually complain of hematuria, like those with renal cell and urothelial carcinomas. It is hard to distinguish between them based upon clinical signs alone. We considered other diseases and found urothelial cell carcinoma in the bladder. Renal SmCC shares comparable morphological and immunohistological features with SmCC of other organs, and renal SmCCs often accompany urothelial carcinomas, suggesting a potential association between $\mathrm{SmCC}$ and urothelial carcinoma in the kidney [7]. An immunohistochemical examination was performed in all samples. Our patient's specimens of kidney and lung were strongly positive for CD56, synaptophysin and TTF-1, and weakly positive for chromogranin. Although there is no lung specific tumor marker, with the help of a relatively restricted marker, TTF-1, it is possible to separate a lung primary from a metastasis with a reasonable degree of certainty [8]. Some extrapulmonary small cell carcinomas were positive for TTF-1, but other extrapulmonary neuroendocrine tumors were not positive for TTF-1. Although small cell carcinomas in the bladder (2/4), uterine cervix $(1 / 7)$ and prostate (4/4) were positive for TTF-1, renal small cell carcinoma was negative $(0 / 1)[9]$.

Hematuria is a common symptom, and we considered all possibilities during the differential diagnosis. Kidney metastasis from lung cancer is rare, and is found in conjunction with bladder cancer even more rarely. In this case, the renal and bladder lesions were detected retrospectively. To prevent unnecessary surgery in such cases, fine needle biopsies should be performed.

\section{Acknowledgments}

None to declare.

\section{Financial Disclosure}

None to declare. 


\section{Conflict of Interest}

None to declare.

\section{Informed Consent}

Informed consent was obtained from the patient for publication of this case report and any accompanying images.

\section{Author Contributions}

Two authors contributed to the writing and correction of this manuscript as well as being involved in the patient treatment.

\section{References}

1. Kim JH, Lee SH, Park J, Kim HY, Lee SI, Nam EM, Park JO, et al. Extrapulmonary small-cell carcinoma: a singleinstitution experience. Jpn J Clin Oncol. 2004;34(5):250254.

2. Becker WE, Schellhammer PF. Renal metastases from carcinoma of the lung. Br J Urol. 1986;58(5):494-498.

3. Cohen MH, Matthews MJ. Small cell bronchogenic carcinoma: a distinct clinicopathologic entity. Semin Oncol. 1978;5(3):234-243.

4. Cai J, Liang G, Cai Z, Yang T, Li S, Yang J. Isolated renal metastasis from squamous cell lung cancer. Multidiscip Respir Med. 2013;8(1):2.

5. Rini BI, Campbell SC, Escudier B. Renal cell carcinoma. Lancet. 2009;373(9669):1119-1132.

6. Honda H, Coffman CE, Berbaum KS, Barloon TJ, Masuda K. CT analysis of metastatic neoplasms of the kidney. Comparison with primary renal cell carcinoma. Acta Radiol. 1992;33(1):39-44.

7. Si Q, Dancer J, Stanton ML, Tamboli P, Ro JY, Czerniak BA, Shen SS, et al. Small cell carcinoma of the kidney: a clinicopathologic study of 14 cases. Hum Pathol. 2011;42(11):1792-1798.

8. Jagirdar J. Application of immunohistochemistry to the diagnosis of primary and metastatic carcinoma to the lung. Arch Pathol Lab Med. 2008;132(3):384-396.

9. Agoff SN, Lamps LW, Philip AT, Amin MB, Schmidt RA, True LD, Folpe AL. Thyroid transcription factor-1 is expressed in extrapulmonary small cell carcinomas but not in other extrapulmonary neuroendocrine tumors. Mod Pathol. 2000;13(3):238-242. 